Robents, C. F. (1959). J. gen. Microbiol. 20, 540-548

\title{
A Replica Plating Technique for the Isolation of Nutritionally Exacting Mutants of a Filamentous Fungus (Aspergillus nidulans)
}

\author{
By C. F. ROBERTS \\ Department of Genetics, The University, Glasgore
}

SUMMARY: This paper describes a replica plating technique for a filamentous fungus and shows its effectiveness in the isolation of nutritionally-exacting mutants, including mutants unable to grow with certain sugars as sole carbon source.

The two main applications of the replica plating technique are in the classification of colonies differing from each other in a number of known nutritional requirements, and in the isolation of new mutant strains. The first application is mainly in experiments on gene recombination where nutritional requirements are used as genetic characters. Organisms derived by recombination are plated so that each can give rise to a colony, and then each of the colonies is classified according to its combination of nutritional characters. It is not usually necessary to test more than a few hundred colonies for their response to a small number of nutrients. A convenient method for doing this ( $\mathbf{M r}$ E. C. Forbes, personal communication) is to prepare initial plates with a number of the colonies, perhaps 30, set in a standard pattern on each plate. These colonies are then replicated on to a series of test media by means of a multi-point inoculator, the points of which are also arranged in the standard pattern. The colonies are classified by their failure to grow on test media lacking particular nutrients. Such an application is now well known and has been used routinely in this laboratory for several years.

In the second application of replica plating, i.e. the isolation of mutants, the preparation of initial plates with an ordered pattern of colonies is impracticable because it is necessary to test many thousands of colonies, since mutants occur very infrequently. Lederberg \& Lederberg (1952) resolved this difficulty by using a velveteen pad to replicate rapidly from initial plates bearing large numbers of randomly distributed colonies. Their technique and its variations have found wide usage in microbiology, and have been successfully applied to bacteria (Lederberg \& Lederberg, 1952), to actinomycetes (Braendle \& Szybalski, 1957) and to unicellular algae (Eversole, 1956).

Lederberg's replica plating technique has not been previously described in a form that can be applied readily to a filamentous fungus. Among the filamentous fungi Aspergillus nidulans has been extensively subjected to detailed genetic investigation (Pontecorvo et al. 1953; Käfer, 1958; Pontecorvo \& Käfer, 1958) and it was desirable to have a straightforward replica plating technique for it. This paper describes the technique developed and its use in isolating nutritionally-exacting mutants of two types. One type are the usual 
auxotrophic mutants which can only grow when a particular nutrient (e.g. an amino acid, vitamin, etc.) is present in the medium. The other type are unable to utilize particular sugars as sole carbon sources for growth and are called 'sugar mutants'.

\section{METHODS}

Design of technique. The general method of replica plating was described by Lederberg \& Lederberg (1952); it is useful to recall here the three essential stages. (1) Spreading a suspension of mainly separate organisms on a solid medium so that after incubation most of the colonies on the initial plate have grown from single organisms. (2) The use of a pad of sterile velveteen or similar rough material (e.g. filter-paper) to make replica inoculations from the initial plate on to a series of plates with different media ('test plates'). (3) Visual inspection of the series of replica plates to compare the responses of individual colonies to different media. There are a number of essential conditions for replica plating and factors which affect the efficiency of the technique. An obvious condition is that the organism should form discrete colonies on agar media. It is an advantage if these colonies are derived from uninucleate cells, and with fungi they should also sporulate reasonably well.

In the preparation of initial plates it is necessary to strike a balance between the number of colonies tested on each plate and the fact that with increased crowding of colonies the proportion of mutants that will not be detected because of mixed growth (syntrophy) also increases. Obviously organisms with nuclei which determine a nutritional requirement will only be detected when growing in a pure clone, and not when mixed with organisms which have unaltered nuclei and consequently are not exacting.

Choice of material for a replica pad is largely governed by the organism. In general a velveteen pad or a filter-paper disk are suitable for wettable spores or vegetative cells, especially bacteria, while dry spores are most effectively transferred on some type of metal prong.

In Aspergillus nidulans uninucleate haploid dry conidia are formed in profusion on solid media and suspensions of mainly separate conidia can easily be obtained. When conidia germinate close together on agar their hyphae tend to grow together and form mixed colonies. However, this does not occur between hyphae from conidia which are more than $0.5 \mathrm{~cm}$. apart, and these conidia give rise to discrete colonies separated by zones of no growth. A Petri dish of $9.5 \mathrm{~cm}$. internal diameter would accommodate $c .300$ colonies if the conidia were at regular intervals of $0.5 \mathrm{~cm}$., but in practice the upper limit, in order to avoid too many mixed colonies, is about 200 conidia on each initial plate.

Velveteen and filter-paper have proved only partly suitable for a replica pad (Dr E. Calef, personal communication). Moreover, with this type of pad only a single inoculum can be taken from the initial plate and this does not allow more than three replicas to be made. Eventually a 'replicator' similar to the multi-point inoculators used for Aspergillus to classify colonies by their nutritional requirements was chosen, but having many more points 
like the instrument for use with Streptomyces mentioned by Braendle \& Szybalski (1957).

Construction of the replicator. One of the replicators is shown in $\mathrm{Pl} .1$, fig. 1. The base-plate is made from Perspex sheet in which holes that allow easy fitting of the steel pins (dressmaker's pins $2.5 \mathrm{~cm}$. long) without letting their heads through are drilled in a regular pattern at $0.5 \mathrm{~cm}$. intervals. It is important that the points of the pins should be in one plane, therefore the pins should be of equal lengths and the base-plate flat. The pins are held in position by Perspex cement, and a reference point to orientate the replica is provided by omitting a few of them from the pattern.

General method of application. To prepare the initial plates a dilute spore suspension is spread on solid medium to yield 100-200 colonies distributed evenly on each plate, and the plates incubated until good sporulation has taken place. The series of test plates are prepared with media chosen according to the purpose of replication (examples below) and dried to remove excess moisture. After sterilizing the replicator by dipping the pins in ethanol and igniting, the first initial plate is opened with the colonies upwards and the replicator lowered on to them. It is then withdrawn, laid on the bench with the pins uppermost and the first test plate inoculated by touching it against the points. The replicator can be recharged without further sterilization, and the sequence is repeated to inoculate each test plate of the series.

It is, of course, essential that all inocula are taken from exactly the same positions on the initial plate. This is readily achieved if the holes made in the initial plate when taking the first inoculum are used as guides for succeeding inoculations. Eight replica plates can be made from one initial plate without difficulty. With attention to normal aseptic routine contamination is negligible.

Strains and media. Strains of Aspergillus nidulans held in the Genetics Department of Glasgow University were used. Media and the general technique for handling $A$. nidulans given by Pontecorvo et al. (1953) were followed unless otherwise stated. Minimal medium (MM) was a solution of inorganic salts, including nitrate and sulphate as main nitrogen and sulphur sources, with $1 \%(\mathrm{w} / \mathrm{v})$ glucose and $1.5 \%$ agar. Complete medium (CM) was a complex medium containing casein hydrolysate, yeast extract, various vitamins, a hydrolysate of yeast nucleic acid and the same concentrations of inorganic salts, glucose and agar as in MM.

\section{RESULTS}

\section{$A$ reconstruction experiment to illustrate the general} application of the technique

Serial dilutions of a mixed suspension of conidia from three strains of Aspergillus nidulans with different conidial colours and nutritional requirements were spread on CM, incubated and plates with suitable numbers of colonies selected as initial plates. The replicator was used to inoculate a series of Petri dishes containing test media which in turn allowed only one of the three 
strains to grow, and finally on to $\mathrm{CM}$ on which they all grew. Plate 1, fig. 2, shows one of the sets of dishes; Table 1 gives details of strains, media and description of the photograph.

Table 1. A reconstruction experiment to illustrate the general application of replica plating to Aspergillus nidulans (see also Pl. 1, fig. 2)

Colonies of the three strains were grown together on the initial plate and replicas made on three test plates, each of which allowed only one of the three strains to grow, and finally on to CM on which they could all grow.

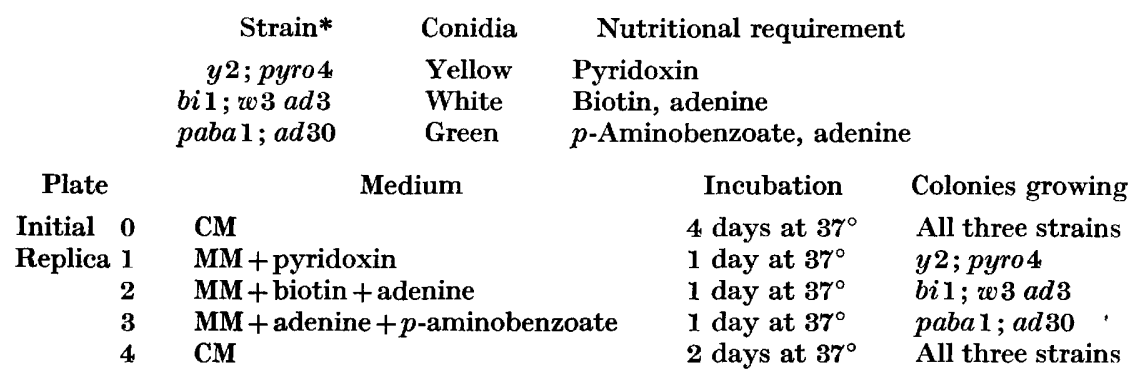

* Symbols of mutant alleles: (a) Requirements, pyro4 = pyridoxin; bi $1=$ biotin; ad 3 and $a d 30=$ adenine (different loci); paba $1=p$-aminobenzoate. (b) Conidial colours, $y \mathbf{2}=$ yellow; $w 3=$ white; the wild type is green. (See Pontecorvo et al. 1953, for further details.)

In Pl. 1, fig. 2, orientation of the replicas is shown by three spots near the lower edge of the initial plate and three corresponding stabs or colonies on the other plates. Green, yellow and white colonies can be distinguished on plates 0 and 4 by their different shades. A number of mixed colonies on plate 4 have grown on more than one of the test plates, while plate 3 shows slight growth of strain bi 1 ; w3 ad3 (point-like colonies) where close to colonies of other strains from which biotin was diffusing into the medium. It is clear from Pl. 1, fig. 2, that colonies with different nutritional requirements can be identified by their different growth responses when replicated on to the appropriate test media. Combination of nutritional requirements with different conidial colours allows the identification to be verified and provides a good illustration of the general application of replica plating to Aspergillus nidulans.

\section{Reconstruction experiments with increasing numbers of colonies on the initial plates}

Reconstruction experiments were done to investigate to what extent the efficiency of the technique decreases with increasing total number of colonies on the initial plates when, as in the actual isolation of mutants, the mutants sought represent a small proportion of all colonies. Mixed platings of two auxotrophic strains were used to simulate the situation when isolating nutritionally-exacting mutants, and to provide an estimate for the optimal density of colonies on initial plates when using the technique for this purpose. The two auxotrophic strains chosen had differently coloured conidia, to facilitate scoring. Suspensions of conidia of Aspergillus nidulans strains $y 2$; pyro 4 and 
w3 ad3 were mixed and plated on CM to give a series of initial plates with c. 10 colonies of $w 3 a d 3$ and 50-500 colonies of $y 2$; pyro 4 on each plate. (See Table 1 for explanation of symbols.) Replication was performed to see how many of the initial w3 ad3 colonies could be identified on the replica plates by their failure to grow on an adenine-deficient test medium. Replicas were therefore made from each initial plate on to $\mathrm{MM}+$ pyridoxin (no adenine) and on to $\mathrm{CM}$ (containing adenine). The test plates were compared after incubation for $24 \mathrm{hr}$. at $37^{\circ}$ and the positions of colonies growing on CM but not on MM + pyridoxin marked. Incubation was continued and the scoring finally checked by inspection of conidial colours on the CM plates. A high proportion of colonies marked after $24 \mathrm{hr}$. as possible colonies of strain $w 3$ ad3 proved to have been scored correctly.

Table 2. Reconstruction experiments to show the loss of efficiency of the replica plating technique as the number of colonies on the initial plates increases

Initial plates were prepared with about 10 colonies of Aspergillus nidulans, strain w3 ad3 and from 50 to 500 colonies of strain $y 2$; pyro4. Replicas were made on to MM + pyridoxin and on to CM and compared after incubation for $24 \mathrm{hr}$. to identify colonies of strain w3 ad3.

\begin{tabular}{|c|c|c|c|}
\hline \multicolumn{4}{|c|}{ Initial plates } \\
\hline \multirow{2}{*}{$\begin{array}{l}\text { No. of } \\
\text { plates }\end{array}$} & \multirow{2}{*}{$\begin{array}{l}\text { Mean } \\
\text { no. of } \\
\text { colonies/ } \\
\text { plate }\end{array}$} & \multicolumn{2}{|c|}{ Total no. of colonies } \\
\hline & & $y_{2 ; \text { pyro4 }}$ & $w 3$ ad 3 \\
\hline 4 & 45 & 139 & 41 \\
\hline 4 & 79 & 290 & 27 \\
\hline 8 & 110 & 798 & 78 \\
\hline 4 & 162 & 612 & 37 \\
\hline $\mathbf{2}$ & 205 & 396 & 14 \\
\hline $\mathbf{3}$ & 305 & 870 & 44 \\
\hline 2 & c. 500 & c. 1000 & 28 \\
\hline
\end{tabular}

$\begin{gathered}\text { No. of colonies } \\ \text { of w3 ads } \\ \text { identified on } \\ \text { the replica } \\ \text { plates }\end{gathered}$
$39(95 \%)$
$21(78 \%)$
$67(86 \%)$
$25(68 \%)$
$6(43 \%)$
$18(41 \%)$
$6(21 \%)$

Further experiments of the same type were performed by replicating directly on to CM and scoring by conidial colour alone. Those colonies which showed only white conidia under the binocular microscope were counted as recoveries. When w3 ad3 colonies on the replica plates formed obvious groups each group was counted as one as it probably represents a single colony on the initial plate. The data are presented in Table 2. It is apparent that as the total number of colonies on the initial plates increased fewer w3 ad3 colonies were identified on the test plates. At a density of 100-200 colonies on each initial plate (the average proportion of the rare component was $\mathbf{0 . 0 6}$ for these plates) identification was of the order of $70 \%$ of the $w 3$ ad3 colonies on the initial plates. In the isolation of nutritional mutants described below, the mutagenic treatment used is known from previous work to yield a proportion of 0.01 auxotrophs among the colonies growing from spores which survive the treatment. Therefore, though the reconstruction experiment does not exactly parallel the conditions used when isolating auxotrophs after mutagenic treatment, the figures indicate that about $70 \%$ of nutritional mutants present on initial plates may be isolated by replica plating. 


\section{Isolation of auxotrophic mutants by replica plating}

Screening experiments were done primarily to isolate sugar mutants, but auxotrophic mutants were isolated at the same time to provide some idea of the efficiency of the replica plating technique and to compare the frequencies of auxotrophic and sugar mutants.

Suspensions in saline of mainly separate conidia of Aspergillus nidulans, strains $y 2$; pyro 4 or $b i 1 ; w 3$, were irradiated under a u.v. lamp until the viable count was only $5 \%$ of the untreated controls, then diluted and plated on CM to yield 100-200 colonies/plate. After incubation for 3 days good sporulation had occurred and replicas were made on to $\mathrm{MM}+$ pyridoxin (or MM+ biotin) and on to CM. Plates were compared after incubation for $\mathbf{2 4} \mathrm{hr}$. and isolates of colonies which failed to grow on $\mathrm{MM}+$ pyridoxin (or biotin) but which grew on CM were made from the CM plates. These isolates were retested and those which failed to grow at all on $\mathbf{M M}+$ pyridoxin (or + biotin) were purified by single colony isolation and classified for nutritional requirement by routine methods (Pontecorvo et al. 1953).

Table 3. Auxotrophic mutants of Aspergillus nidulans isolated by replica plating, grouped into nutritional classes and compared with those obtained by other workers by total isolation

Class of nutrient requirement

Nitrite or ammonia†

Thiosulphate or sulphite $\dagger$

Amino acids

Components of yeast nucleic acid

Vitamins

Not tested

\begin{tabular}{rrrr} 
Replica plating & \multicolumn{2}{c}{ Total isolation* } \\
3 & $5 \%$ & 17 & $18 \%$ \\
1 & $2 \%$ & 7 & $8 \%$ \\
11 & $18 \%$ & 12 & $13 \%$ \\
6 & $10 \%$ & 11 & $12 \%$ \\
17 & $31 \%$ & 22 & $23 \%$ \\
20 & $34 \%$ & 24 & $26 \%$ \\
58 & \multicolumn{3}{c}{$\%$} \\
6422 colonies on & 5408 colonies \\
initial plates & tested
\end{tabular}

* Data from Pontecorvo et al. (1953). The figures are not strictly comparable as different starting strains were used and they include strains obtained after irradiation with X-ray, and with u.v. radiation at survival rates other than $5 \%$.

$\uparrow$ The discrepancy between the two methods here is probably due to the 20 partial mutants obtained by replica plating which were not classified. Mutants unable to utilize nitrate or sulphate characteristically give partial growth on MM.

In this experiment 110 isolates were made from 49 initial plates bearing a total of 6422 colonies (4033 of strain $y 2 ;$ pyro 4 and 2389 of strain bi1; w3). After retesting the yield was 58 mutants $(0.9 \%)$ with a new nutritional requirement (36 from strain $y 2 ;$ pyro 4 and 22 from strain $b i 1 ; w 3$ ). Twenty of these mutants were not tested further because the additional requirements for growth were only partial. The absolute requirements of the remaining 38 strains $(0.6 \%)$ are given in the second column of Table 3 , grouped by nutritional classes.

In a second experiment with strain $b i 1 ;$ w 3 only auxotrophic mutants with a total nutritional requirement were isolated and the yield was therefore 
diminished, namely, 36 auxotrophs from c. 7400 colonies tested $(0.5 \%)$. These auxotrophs have not been tested for their additional nutrient requirements.

\section{The isolation of sugar mutants by replica plating}

It was thought worth examining the possibility that strains of Aspergillus nidulans might occur which are unable to use certain sugars as sole carbon source. The screening experiments were carried out in conjunction with the isolation of auxotrophic mutants described above. Colonies on the initial plates were replicated on to series of seven test plates; one was minimal medium $+1 \%(\mathrm{w} / \mathrm{v})$ glucose and the growth factors required by parent strain (the control), five were similar media but with glucose replaced by $1 \%$ galactose, lactose, maltose, sorbitol or sucrose, respectively; a final plate was complete medium. The auxotrophic mutants were detected by failure to grow on the control plate though growing on CM. The sugar mutants were detected by failure to grow on one or more of the five plates with the unusual sugars, though growing on the control plate (with glucose), the remaining sugars, and on CM. Strains presumed to be sugar mutants were isolated from the control plates, retested and purified by single colony isolation. The pure strains were finally tested by plating suspensions of separate conidia on control plates with glucose, and on media with the glucose replaced by the given sugar as commercially supplied or by this sugar twice recrystallized. Only those strains were retained which gave parent growth with glucose as carbon source but little or no growth with the sugar (as received or recrystallized) when colonies arose from single conidia.

Table 4. Sugar mutants in Aspergillus nidulans isolated by replica plating

The sugar mutants were isolated by replica plating and were detected by their failure to grow on a given sugar as sole carbon source.

$\begin{array}{lcc}\text { Carbon source } & \begin{array}{c}\text { No. of } \\ \text { mutants }\end{array} & \begin{array}{c}\text { Minimum } \\ \text { no. of loci }\end{array} \\ \text { Sucrose } & 0 & - \\ \text { Maltose } & 2 & 1 \\ \text { Lactose } & 9 & 1 \\ \text { Galactose } & 8 & 1 \\ \text { Sorbitol } & 7 & 2 \\ \text { Fructose* } & 3 & 1 \\ & 29 & 6\end{array}$

* Mutants first isolated because of their failure to grow on sucrose or sorbitol.

In the experiments with Aspergillus nidulans strains $y 2 ;$ pyro 4 and bi 1 ; w 3 9 sugar mutants $(0 \cdot 14 \%)$ were isolated from 6422 colonies tested by replica plating. In the second experiment with strain $b i 1 ; w 320$ sugar mutants $(0.27 \%)$ were isolated from $c .7400$ colonies. The distribution of these mutants is shown in Table 4, together with the number of loci that they are so far known to involve. The frequency of sugar mutants of types detectable by the tests in the two screening experiments was thus $29 / 13,800(0 \cdot 2 \%)$. In the same 
experiments the frequency of auxotrophic mutants with a clear-cut nutritional requirement was $74 / 13,800(0.5 \%)$. One or more mutants have been obtained for each of the sugars tested, except sucrose.

\section{DISCUSSION}

This paper describes the application of a replica plating technique to a filamentous fungus for the isolation of nutritionally-exacting mutants. The efficiency of the technique in recovering auxotrophic mutants of Aspergillus nidulans is about the same as that of the much more laborious method of total isolation (Pontecorvo et al. 1953). There should not be any difficulty in adapting the technique for work with other filamentous fungi. The main advantage of replica plating in the isolation of mutants is the saving of time and labour which enables a far larger population to be tested in a given time than could be done by the total isolation method. However, replica plating cannot be readily employed for quantitative studies and when an estimate of the numbers of a rare component in a population is required the total isolation method is preferable because every colony obtained after plating is known to have been tested once. In both replica plating and total isolation a fraction of the rare component may not be detected because of the growth of mixed colonies.

The replica plating procedure adopted for Aspergillus nidulans yielded auxotrophic mutants at the rate of $0 \cdot 5-0.9 \%$ of the colonies growing from conidia after $95 \%$ killing by u.v. radiation; yields of the same order are obtained by total isolation after similar u.v. treatment. Reconstruction experiments to test the effectiveness of the procedure indicate a recovery of $c .70 \%$ of colonies of the rarer type used on the initial plates. The procedure appears, therefore, to isolate almost as high a proportion of auxotrophic mutants as would the total isolation method under the same conditions. The distribution of mutants among the major nutritional classes does not show any obvious differences between the two methods, though the numbers investigated were small.

The author wishes to thank Professor G. Pontecorvo, F.R.S., for advice and criticism in the work and in the preparation of this paper, Mr E. C. Forbes for introduction to the techniques for Aspergillus nidulans, and Dr E. R. B. Shanmugasundaram for classifying the auxotrophic mutants. This work is part of a programme supported by the Nuffield Foundation.

\section{REFERENCES}

Braendle, D. H. \& Szybalski, W. (1957). Genetic interaction among streptomycetes: heterokaryosis and synkariosis. Proc. nat. Acad. Sci., Wash. 43, 947.

Eversole, R. A. (1956). Biochemical mutants of Chlamydomonas reinhardi. Amer. J. Bot. 43, 404.

KÄFER, E. (1958). An 8-chromosome map of Aspergillus nidulans. Advanc. Genet. 9, 105.

LederberG, J. \& Lederberg, E. M. (1952). Replica plating and indirect selection of bacterial mutants. J. Bact. 63, 399 . 
Pontecorvo, G. \& KäFER, E. (1958). Genetic analysis based on mitotic recombination. Advanc. Genet. 9, 71.

Pontecorvo, G., Roper, J. A., Hemmons, L. M., Macdonald, K. D. \& Bufton, A. W. J. (1953). The genetics of Aspergillus nidulans. Advan. Genet. 5, 141.

Fig. 1. The replicator.

\section{EXPLANATION OF PLATE}

Fig. 2. Illustration of the application of replica plating to Aspergillus nidulans. Three strains with different nutritional requirements and with green, yellow or white conidia were grown together on the initial plate (0). Replicas were made on test media which allowed the strains with yellow (1), with white (2) or with green conidia (3) to grow. The last plate (4) contained medium on which all three strains grew. Colonies with differently coloured conidia can be distinguished on plates 0 and 4. Plates 1-3 distinguished colonies by their nutritional requirements and the correlation of conidial colour with nutritional requirement can be seen. (Table 1 gives details of the strains and media used.)

(Received 21 November 1958) 
Journal of General Microbiology, Vol. 20, No. 3

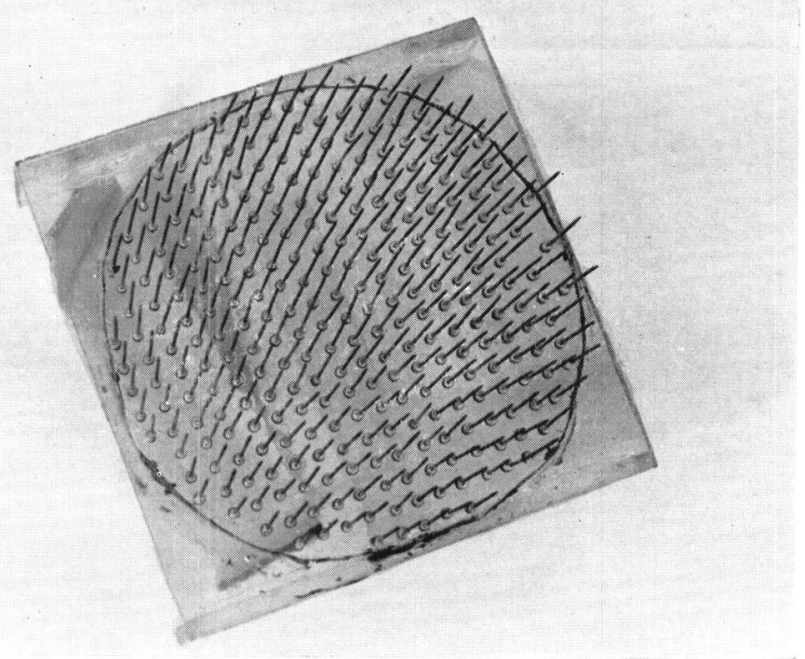

Fig. 1

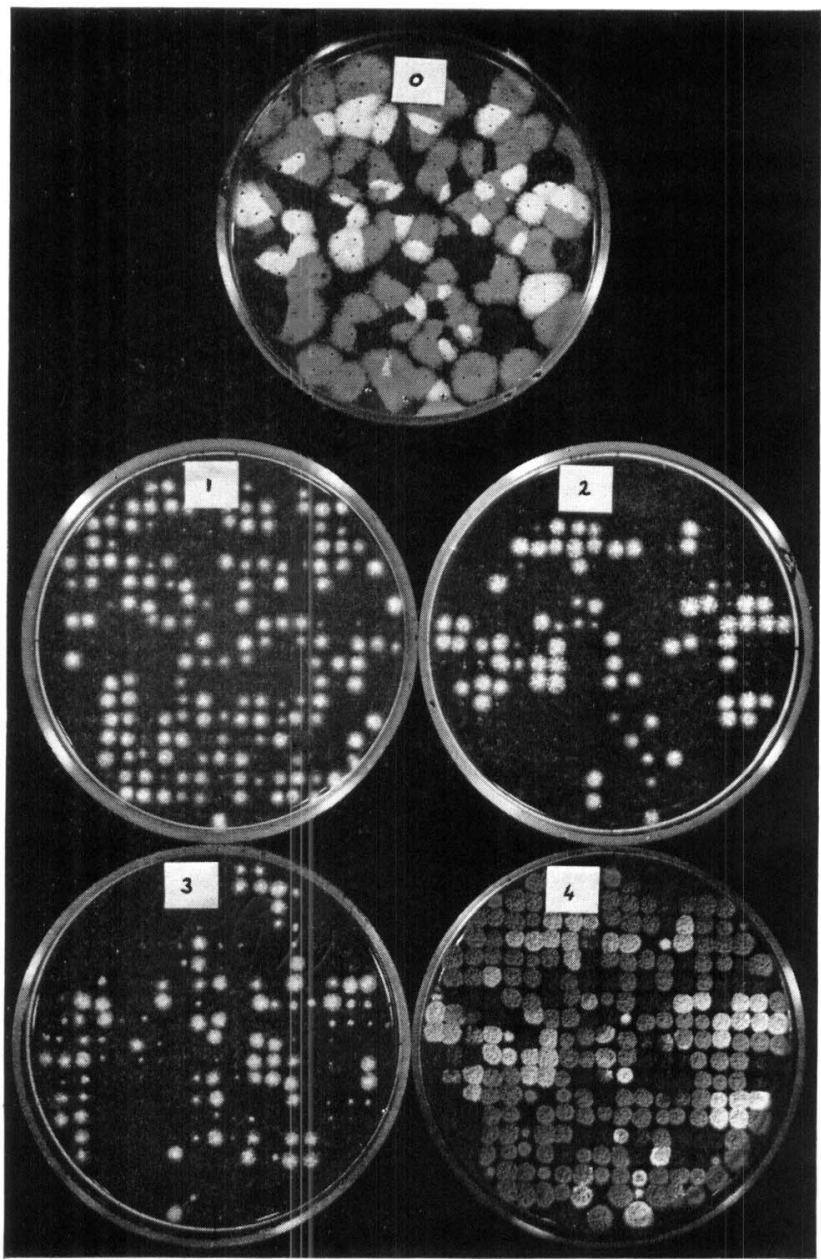

Fig. 2

C. F. Roberts-A replica plating technique. Plate 1 